\title{
Combined effect of frustration and dimerization in ferrimagnetic chains and square lattices
}

\author{
Aiman Al-Omari: and A. H. Nayyar \\ Department of Physics, \\ Quaid-i-Azam University, \\ Islamabad, Pakistan 45320
}

(May 12, 2000)

\begin{abstract}
Within the zero-temperature linear spin-wave theory we have investigated the effect of frustration and dimerization of a Heisenberg system with alternating spins $s_{1}$ and $s_{2}$ on one- and two-dimensional lattices. The combined effect most visibly appears in the elementary excitation spectra. In contrast to the ground state energy that decreases with dimerization and increases with frustration, the excitation energies are shown to be suppressed in energy by both dimerization and frustration. The threshold value of frustration that signals a transition from a classical ferrimagnetic state to a spiral state, decreases with dimerization, showing that dimerization further helps in the phase transition. The correlation length and sublattice magnetization decrease with both dimerization and frustration indicating the destruction of the long-range classical ferrimagnetic. The linear spin wave theory shows that in the case of a square lattice, dimerization initially opposes the frustration-led transition to a spiral magnetic state, but then higher magnitudes of lattice deformation facilitate the transition. It also shows that the transition to spiral state is inhibited in a square lattice beyond a certain value of dimerization.
\end{abstract}

PACS numbers: 75.10.Jm, 75.50.Ee 


\section{Introduction}

Heisenberg ferrimagnetic spin systems, also known as alternating or mixed spin systems, consisting of two sublattices with spins $s_{1}$ and $s_{2}$ of unequal magnitudes with a net nonzero spin per unit cell have received considerable attention recently. Several theoretical studies have been carried out to calculate the ground-state properties and the low-lying excited states of

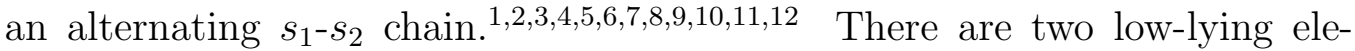
mentary excitations, the gapless ferromagnetic spin wave excitations and the antiferromagnetic spin waves with a gap. Dimerization of the lattice has been shown to lower the ground-state energy, sublattice magnetization and excitation energies of a ferrimagnetic system. 13

Frustration due to competing antiferromagnetic second neighbor interactions can in principle destroy any LRO of the Neel type. Ivanov et al. used spin wave expansion, density matrix renormalization group, and an exact diagonalization technique to investigate the effect of weak frustration on the ground state energy of a quantum Heisenberg ferrimagnetic chain. They have identified several critical frustration parameters. The first, called $\alpha_{c}$, heralds a transition from the classical commensurate ferrimagnetic state to a spiral state. The second special point, called $\alpha_{D}$ and termed as the disorder point marks the onset of incommensurate finite-range spin-spin correlations. The third special point, called $\alpha_{T}$, is a first order transition point from the long-range ordered ferrimagnetic state with total spin $S_{g}=N\left(s_{1}-s_{2}\right)$ to a singlet state with $S_{g}=0$. They found that frustration causes an increase in the ground state energy.

In classical ferrimagnets, one hopes to see two transition points resulting from frustration: one where the order is completely destroyed, and the other where the classical ferrimagnetic state changes into a spiral state.

The objective of the present study is two-fold: to investigate these transitions in a square lattice ferrimagnet, and to study the combined effect of dimerization and frustration. The purpose of the latter exercise would be to see the effect of dimerization on the transitions induced by frustration. For this purpose, we will study systems with $\left(s_{1}, s_{2}\right)$ equal to $\left(1, \frac{1}{2}\right),\left(\frac{3}{2}, 1\right)$ and $\left(\frac{3}{2}, \frac{1}{2}\right)$ using a zero temperature linear spin wave (LSW) theory. 14 The 
choice of the spins is guided by the recent assertion that the three systems have different predominant characters: the first has a mixed ferromagnetic and antiferromagnetic character, the second is more antiferromagnetic and the third is more ferromagnetic in character.10,11,12 Validity of the linear spin wave theory will be a serious question for frustrated systems, but it has already been argued by Ivanov et al. that the LSW theory yields satisfactory results, at least for small values of frustration.

In order to set the background for the combined effect of dimerization and frustration on square lattice, we shall first use the LSWT to investigate the effect in ferrimagnetic chains. Section II below sets up the LSW formalism for a dimerized ferrimagnetic chain in the presence of frustration. It has already been argued 5 in discussing dimerization of square lattices that a proper account of various possible dimerized configurations as a result of lattice distortions can be taken only when the nearest neighbor interaction is taken as $J(r) \sim \frac{J}{r}$. This choice made it possible to conclude that among the various possibilities, plaquette configuration had the lowest ground state energy. But this form of the nearest neighbor interaction has its own consequences, and we shall study those for the chains also in Section II in order to identify its particular effects to distinguish them from the effects of dimensionality of the lattice. Effects of frustration on a dimerized square lattice system are then studied in section III.

\section{Ferrimagnetic chains with dimerization and frus- tration}

Mixed spin chain systems have recently been studied extensively within

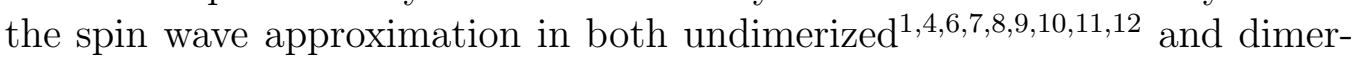
ized 3 - regimes. It has been conclusively shown that the linear spin wave theory gives excellent results for the ground state energy and magnetization of ferrimagnets. We consider a chain consisting of two sublattices occupied by spins $s_{1}$ and $s_{2}\left(s_{1}>s_{2}\right)$ allowing for both intersublattice and intrasublattice nearest neighbor interactions $J_{1}$ and $J_{2}$ respectively. We choose to describe this system by the Hamiltonian 


$$
H=\sum_{n}\left[J^{+} \mathbf{S}_{1, n} \cdot \mathbf{S}_{2, n}+J^{-} \mathbf{S}_{2, n} \cdot \mathbf{S}_{1, n+1}+J_{2}\left(\mathbf{S}_{1, n} \cdot \mathbf{S}_{1, n+1}+\mathbf{S}_{2, n} \cdot \mathbf{S}_{2, n+1}\right)\right],
$$

where $J^{ \pm}=J_{1}(1 \pm \delta)$, and $\delta$ is the dimerization parameter that varies from 0 to 1 . The total number of sites (or bonds) is $2 N$ and the sum is over the $N$ unit cells.

The usual boson representation of spin operators in the two sublattices is

$$
\begin{aligned}
& S_{1, n}^{+}=\left(2 s_{1}-a_{n}^{\dagger} a_{n}\right)^{1 / 2} a_{n}, S_{2, n}^{+}=b_{n}^{\dagger}\left(2 s_{2}-b_{n}^{\dagger} b_{n}\right)^{1 / 2}, \\
& S_{1, n}^{z}=s_{1}-a_{n}^{\dagger} a_{n}, \quad S_{2, n}^{z}=b_{n}^{\dagger} b_{n}-s_{2},
\end{aligned}
$$

In terms of the normal mode operators

$$
\begin{aligned}
& \alpha_{k}=u_{k} a_{k}-v_{k} b_{k}^{\dagger}, \\
& \beta_{k}=u_{k} b_{k}-v_{k} a_{k}^{\dagger},
\end{aligned}
$$

the linearized Hamiltonian in Eq. (1) becomes

$$
\tilde{H}=\varepsilon_{g}+\sum_{k}\left[E_{1}(k) \alpha_{k}^{\dagger} \alpha_{k}+E_{2}(k) \beta_{k}^{\dagger} \beta_{k}\right],
$$

The ground-state energy per unit cell $\varepsilon_{g}$ and the energies of the two excitation modes $E_{1}(k)$ and $E_{2}(k)$ are given by

$$
\begin{aligned}
& \varepsilon_{g}=C-\sum_{k}\left[A_{1}(k)+A_{2}(k)-\xi(k)\right], \\
& E_{1}(k)=\frac{1}{2}\left(A_{1}(k)-A_{2}(k)+\xi(k)\right), \\
& E_{2}(k)=\frac{1}{2}\left(A_{2}(k)-A_{1}(k)+\xi(k)\right) .
\end{aligned}
$$

In these equations

$$
\begin{aligned}
\xi_{k} & =\sqrt{\left(A_{1}(k)+A_{2}(k)\right)^{2}-4 B^{2}(k)} \\
A_{1}(k) & =J_{p} s_{2}-\alpha s_{1}[1-\cos (2 k)] \\
A_{2}(k) & =J_{p} s_{1}-\alpha s_{2}[1-\cos (2 k)] \\
B(k) & =\sqrt{s_{1} s_{2}} \Lambda_{k} \\
\Lambda_{k} & =J_{p} \sqrt{\cos ^{2}(k)+\delta^{2} \sin ^{2}(k)} \\
C & =-J_{p} s_{1} s_{2}+\frac{\alpha}{2}\left(s_{1}^{2}+s_{2}^{2}\right)
\end{aligned}
$$


where $J_{p}=\frac{1}{2}\left(J^{+}+J^{-}\right)$and $\alpha=\frac{J_{2}}{J_{1}}$ is the frustration parameter.

The coefficients $u(k)$ and $v(k)$, constrained by the condition $u^{2}(k)-$ $v^{2}(k)=1$, are given by

$$
\begin{aligned}
& u(k)=\sqrt{\frac{A_{1}(k)+A_{2}(k)+\xi(k)}{2 \xi(k)}}, \\
& v(k)=\sqrt{\frac{A_{1}(k)+A_{2}(k)-\xi(k)}{2 \xi(k)}},
\end{aligned}
$$

Staggered magnetization in the two sublattices corresponding to the spins $s_{1}$ and $s_{2}$, respectively, is

$$
\begin{aligned}
& M_{1}=S_{1}-<D> \\
& M_{2}=<D>-S_{2}
\end{aligned}
$$

where $<D>=<a_{k}^{\dagger} a_{k}>=<b_{k}^{\dagger} b_{k}>$ is the average taken in the ground state, which is the Neel-like state at zero temperature:

$$
<D>=\frac{1}{N} \sum_{k} v^{2}(k)
$$

with $k$ running from $-\frac{\pi}{2}$ to $\frac{\pi}{2}$ which is the first reduced Brillouin zone.

For a two-spin system, we can think of three types of spin-spin correlation functions; $\left.<S_{1,0}^{z} \cdot S_{1, n}^{z}\right\rangle,<S_{2,0}^{z} \cdot S_{2, n}^{z}>$ and $\left\langle S_{1,0}^{z} \cdot S_{2, n}^{z}\right\rangle$. We are interested in the antiferromagnetic correlations which we define as

$$
\begin{aligned}
C_{n} & \equiv<S_{1,0}^{z} \cdot S_{2, n}^{z}>-<S_{1,0}^{z}>\cdot<S_{2, n}^{z}> \\
& =-<O>^{2}
\end{aligned}
$$

where

$$
<O>=\frac{1}{N} \sum_{k} \cos (k n) \cdot u(k) \cdot v(k)
$$

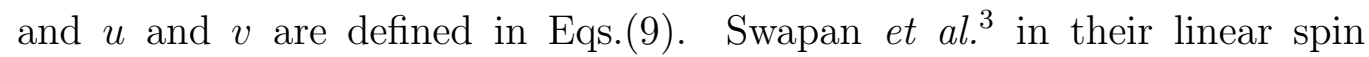
wave analysis when fit this correlation function to $e^{-r / \xi}$ found the inverse correlation length $\xi^{-1}=\ln \frac{s_{1}}{s_{2}}$. For $\left(s_{1}, s_{2}\right)=\left(1, \frac{1}{2}\right)$, this gives $\xi=1.44$, 
whereas their variational calculation gives $\xi=0.75$. Others 6 fit it to the Ornstein-Zernike form

$$
C(r) \sim \frac{e^{-r / \xi}}{\sqrt{r}}
$$

and found it to be 1.01 .

Results of the spin wave theory of ferrimagnets have been discussed ear-

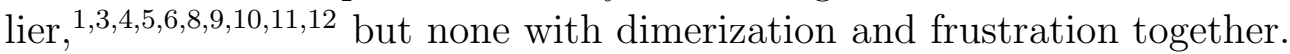

There is a critical value of the frustration parameter $\alpha$ in the linear spin wave theory at which the energies do not remain real, signaling destruction of the long range order. This critical value, that we call $\alpha_{c}$, is strongly $\delta$-dependent, as shown in Fig.(1). At $\delta=0, \alpha_{c}=\frac{s_{1}}{2\left(s_{1}+s_{2}\right)}$. For a $\left(1, \frac{1}{2}\right)$ chain this is $1 / 3$, whereas earlier DMRG results 6 gave $\alpha_{c}=0.28$.

It is already known that with $J^{ \pm}=J_{1}(1 \pm \delta)$ the ground state energy decreases with $\delta$ and scales as $\delta^{2} .615$ It however has a more interesting behavior with respect to $\alpha$. As shown in Fig.(2), the ground state energy per site initially increases with $\alpha$ and then decreases before the long range order is destroyed by frustration at $\alpha_{c}$. This is true even when there is no dimerization, where the results agree with those of Ivanov et al.6 who give values only up to where the maximum occurs. The maximum shifts to lower values of $\alpha$ with $\delta$ as $\delta^{2}$. The curves in Fig.(2) terminate at $\alpha_{c}$ for the corresponding $\delta$. This behavior is true for all the three spin systems considered.

With two atoms per unit cell, a ferrimagnet has to have two modes of elementary excitations. The acoustic mode is gapless $\left(E_{1}(k=0)=0\right)$ and has ferromagnetic character while the optic mode $E_{2}(k)$ is antiferromagnetic and has a gap at $k=0$.

Both acoustic and optic excitation mode energies decrease as $\delta$ increases, as they also do when $\alpha$ increases. This behavior is shown in Fig.(3). The two excitation modes in all the three spin systems scale with $\delta$ as $\delta^{2}$ and linearly with $\alpha$. There is a critical value of $\alpha$ at which the elementary excitation modes start to soften, signaling a transition from a Neel-like spin structure to a spiral structure. 6 This critical value, that we call $\alpha^{*}$ and evaluate from the changing signs of the slopes of the dispersion curves, is different for the acoustic and optic modes, and in the presence of dimerization is $\delta$-dependent: 


$$
\begin{gathered}
\alpha_{\text {acoustic }}^{*}=\frac{s_{1} s_{2}}{2\left(s_{1}^{2}+s_{2}^{2}\right)} J_{p}\left(1-\delta^{2}\right) \\
\alpha_{\text {optic }}^{*}=\frac{1}{4} J_{p}\left(1-\delta^{2}\right)
\end{gathered}
$$

For $\delta=0$, the first of these reproduces the critical value reported by Ivanov et al 6 (denoted therein as $\alpha_{c}$ ). A uniform decrease of $\alpha^{*}$ with $\delta$ leads one to conclude that the transition to spiral spin state caused by frustration is facilitated by dimerization. The spin wave theory gives different behavior of mode softening in the two elementary excitation modes; in the case of the ferromagnetic mode, the mode softening starts at an $\alpha$ that depends upon the magnitudes of the two component spins while in the case of the antiferromagnetic mode it is uniform for all the pairs of the ferrimagnetforming spins.

The magnetization of the two sublattices, as given by Eq.(10), decreases in magnitude with both $\delta$ and $\alpha$ as shown in Fig.(4). The decrease with $\alpha$ indicates the destruction of magnetic order.

The spin-spin correlations decay rapidly with the spin-spin separation, as noted earlier also.6 When fit to the Ornstein-Zernike from, Eq.(15), the correlation length is also found to decrease with both $\delta$ and $\alpha$, as shown in Fig. (5).

The case of $J^{ \pm}=\frac{J_{1}}{1 \mp \delta}$

We shall now look at the same results in the event the spin-spin interaction amplitude is taken to vary inversely with distance between the spins. It has been argued earlier that the choice of nearest neighbor interaction as $J(1 \pm \delta)$ did not allow taking into account the several spin-Peierls distortions possible in a square lattice. A more general choice we proposed was $J(r) \sim \frac{J}{r}$. In the case of nearest neighbor coupling, this means that the amplitudes $J^{ \pm}$ in Eq.(11) are $J^{ \pm}=\frac{J_{1}}{1 \mp \delta}$ which approximate to the more familiar $J_{1}(1 \pm \delta)$ in the limit of small $\delta$. We shall in the following describe the results for a ferrimagnetic chain when the proposed interaction is taken into account.

The critical value $\alpha_{c}$ now has a completely different dependence on $\delta$. For all the three ferrimagnetic systems, it remains constant for most of the $\delta$-range, but increases rapidly at higher values of $\delta$, as shown in Fig.(6). This implies that in this case dimerization does not facilitate destruction of long range order by frustration until it is rather large in magnitude. This 
manifests in the variation of the ground state energy with $\delta$ and $\alpha$, as shown in Fig.(7). Since at $\delta=0$, the two cases of $J^{ \pm}$coincide, therefore $\alpha_{c}$ at $\delta=0$ is the same for both and is $\frac{s_{1}}{2\left(s_{1}+s_{2}\right)}$.

The difference shows up in $\alpha^{*}$ also. In this case $J_{p}=\frac{1}{1-\delta^{2}}$ in Eq.(16), because of which $\alpha^{*}$ for both acoustic and optic modes becomes $\delta$-independent. This contrast between the two choices of $J^{ \pm}$may in fact be taken to be a strong point that could decide, if ever tested, which of the two forms must be chosen to represent dimerization.

The ground sate energy exhibits the same general $\delta$ - and $\alpha$-dependence as in the case of the coupling $J(1 \pm \delta)$. Dimerization reduces the energy and frustration increases it, the reduction by dimerization being larger than the increase by frustration. The increase with frustration is again up to a certain value of $\alpha$ after which the ground state energy shows a decline before $\alpha_{c}$ is approached. This value of $\alpha$ shifts to the lower side as $\delta$ increases, disappearing almost completely in the $\delta \rightarrow 1$ limit as shown in Fig.(7). The difference is in the way in which the ground state energy scales with the two parameters. For $\alpha=0$, it scales with $\delta$ as $\frac{\delta^{1.5}}{|\ln \delta|}$, as reported earlier, lind $^{2}$ and $\delta=0$ it scales with $\alpha$ as $\alpha^{0.5}$.

The dependence of the excitation modes on $\delta$ and $\alpha$ is also quite in contrast to that for the usual case of $J(1 \pm \delta)$ coupling. Frustration continues to suppress the excitation mode energies. On the other hand, while for $\alpha<\alpha^{*}$, an increase in $\delta$ pushes up both the excitation energies, for $\alpha \geq \alpha^{*}$, the acoustic and optic modes differ in their dependence on $\delta$ : the acoustic mode is further suppressed by an increasing $\delta$, but the optic mode is pushed up. This behavior is illustrated in Fig.(8). Note also that the antiferromagnetic mode at $k=0$ depends upon $\delta$ but not on $\alpha$.

Sublattice magnetization and correlation lengths show the same schematic decreasing behavior with $\delta$ and $\alpha$ as in the case of the interaction $J(1 \pm \delta)$, except for the effect of the peculiar dependence of $\alpha_{c}$ on $\delta$ [Fig.(6)].

\section{Frustration on a square lattice}

There are several ways in which a two-dimensional lattice can be deformed in dimerization. This was discussed in detail earlier 5 where it was shown 
that among the various possibilities, the plaquette configuration is the lowest energy deformation. To arrive at this conclusion it was necessary to take the nearest neighbor spin-spin interactions to depend upon the spin-spin distance $r$ as $\frac{J}{r}$. The consequences of this for a chain have been discussed above. In studying the combined effect of dimerization and the competing second neighbor interactions on a square lattice, it becomes imperative to work with this form of interaction.

Since it has already been established that among the possible deformations of a square lattice, the one that involves two phonons, with wavevectors $(\pi, 0)$ and $(0, \pi)$, forming a plaquette lattice, is energetically the most favorable one, 5 , 17 we will restrict our investigation to this kind of deformation alone.

We will write the Hamiltonian of a ferrimagnetic square lattice as a sum of the nearest neighbor and the next nearest neighbor (or intersublattice and intrasublattice nearest neighbor) parts:

$$
\begin{aligned}
H & =H_{1}+H_{2} \\
H_{1} & =\sum_{i, j}^{\sqrt{N}} \sum_{\lambda= \pm 1} J_{\lambda}\left[\mathbf{S}_{1, i, j} \cdot \mathbf{S}_{2, i+\lambda, j}+\mathbf{S}_{1, i, j} \cdot \mathbf{S}_{2, i, j+\lambda}\right] \\
H_{2} & =\sum_{i, j}^{\sqrt{N}} \sum_{\lambda, \lambda^{\prime}= \pm 1} J_{\lambda, \lambda^{\prime}}\left[\mathbf{S}_{1,2 i, 2 j} \cdot \mathbf{S}_{1,2 i+\lambda, 2 j+\lambda^{\prime}}+\mathbf{S}_{2,2 i, 2 j} \cdot \mathbf{S}_{2,2 i+\lambda, 2 j+\lambda^{\prime}}\right]
\end{aligned}
$$

with $J_{\lambda}=\frac{1}{(1-\lambda \delta)}$, and

$$
\begin{aligned}
J_{1,1} & =J_{-1,-1}=\frac{1}{\sqrt{2\left(1+\delta^{2}\right)}} \\
J_{-1,1} & =\frac{1}{\sqrt{2}(1+\delta)} \\
J_{1,-1} & =\frac{1}{\sqrt{2}(1-\delta)}
\end{aligned}
$$

The linear spin wave analysis follows the same procedure as for the chain above. The same equations are applicable in this case, except that the various coefficients have now the following definitions: 


$$
\begin{aligned}
A_{1}(k) & =2 J_{p} s_{2}-\frac{\alpha}{8}\left\{\zeta_{1}^{(1)}\left(J_{1,1}+J_{-1,1}\right)+\zeta_{-1}^{(1)}\left(J_{1,1}+J_{1,-1}\right)\right\} \\
A_{2}(k) & =2 J_{p} s_{1}-\frac{\alpha}{8}\left\{\zeta_{1}^{(2)}\left(J_{1,1}+J_{-1,1}\right)+\zeta_{-1}^{(2)}\left(J_{1,1}+J_{1,-1}\right)\right\} \\
B(k) & =\Gamma(k) \sqrt{s_{1} s_{2}} \\
C & =-2 J_{p} s_{1} s_{2}+\frac{1}{2} \alpha\left(s_{1}^{2}+s_{2}^{2}\right), \\
\Gamma(k) & =\sqrt{J_{p}^{2}\left(\cos \left(k_{x}\right)+\cos \left(k_{y}\right)\right)^{2}+J_{m}^{2}\left(\sin \left(k_{x}\right)+\sin \left(k_{y}\right)\right)^{2}}
\end{aligned}
$$

where

$$
\begin{aligned}
& J_{p}=\left(J_{+1}+J_{-1}\right) / 4=\frac{1}{2\left(1-\delta^{2}\right)} \\
& J_{m}=\left(J_{+1}-J_{-1}\right) / 4=\delta \cdot J_{p} \\
& \zeta_{\sigma}^{(\tau)}=2 \cdot s_{\tau}\left[1-\cos \left(k_{x}+\sigma k_{y}\right)\right] ; \tau=1,2 \text { and } \sigma= \pm 1 .
\end{aligned}
$$

The ground state energy per site $\varepsilon_{g}$ defined in Eq.(5), energies of the two excitation modes $E_{i}(k)$ in Eqs.(6) and (7), staggered magnetization $M_{i}$ defined in Eqs.(10) and correlation length defined in Eq.(15) can now be calculated as functions of the dimerization parameter $\delta$ and frustration parameter $\alpha$. Setting $\alpha=0$ we reproduce the results for unfrustrated dimerized ferrimagnetic square lattice

The linear spin wave theory shows that, like the chain, the ground-state energy of a square lattice decreases with $\delta$ and increases with $\alpha$. As reported earlier, 5 an unfrustrated ferrimagnetic square lattice has a dependence of its ground state energy on $\delta$ as $\frac{\delta^{1.5}}{\ln (\delta) \mid}$. We now also find that ground state energy scales as $\alpha^{0.5}$ for any fixed value of dimerization. This is true for all pairs of spins forming the ferrimagnet.

The elementary excitation spectra are plotted for the system $\left(1, \frac{1}{2}\right)$ in Fig.(9) along the principal symmetry directions in the irreducible Brillouin zone. The same schematic dispersion relations were found for the other two systems. The acoustic and optic modes again have ferromagnetic and antiferromagnetic characters respectively, and both of them are pushed up by dimerization and pulled down by frustration. The optic mode at $\mathbf{k}=(0,0)$ is $\delta$ dependent.

As in the chains, the competing second neighbor interaction also causes a transition from a Neel-like state to a spiral state, indicated by softening 
of the excitation modes. $\alpha^{*}$, the critical value at which the transition takes place, in the case of square lattice is also $\delta$-dependent:

$$
\alpha^{*}=\frac{s_{1} s_{2}}{\sqrt{2}\left(s_{1}^{2}+s_{2}^{2}\right)} \cdot\left(1+\delta^{2}\right)\left(2-\delta^{2}\right)
$$

This is different from the $\alpha^{*}$ in chains on two counts: it is the same for both ferromagnetic and antiferromagnetic modes, and even when the interaction is $J(r)=\frac{J}{r}$, it is $\delta$-dependent. This relation also shows that for $\delta=0$, the value of $\alpha^{*}$ for a square lattice is $2 \sqrt{2}$ times larger than that for a chain. Moreover, unlike a monotonically decreasing $\alpha^{*}$ for a chain, it is a function that is peaked towards higher values of $\delta$, as shown in Fig.(10). This indicates that while the transition to spiral state in a square lattice is initially opposed by dimerization, it is facilitated at larger magnitudes of lattice deformation. This turn around in behavior occurs at $\delta=\frac{1}{\sqrt{2}}$.

The variation of $\alpha^{*}$ and $\alpha_{c}$ with $\delta$ brings out an interesting aspect peculiar to a square lattice ferrimagnet. As shown in Fig.(10), there is a value of $\delta$ at which $\alpha_{c}$ and $\alpha^{*}$ are equal, and the systems which have distorted with $\delta$ beyond this value, which we call $\delta^{*}$, the destruction of order occurs before the onset of spiral magnetic order. The value of $\delta^{*}$ is different for different ferrimagnetic systems.

The sublattice magnetization $M_{i}$ decreases with both $\alpha$ and $\delta$ as shown in Fig.(11). For a non-dimerized square lattice the magnetization has a logarithmic power law scaling behavior with the frustration parameter: $\frac{\alpha^{1.5}}{|\ln \alpha|}$. The same scaling law was found for a dimerized plaquette.

The correlation function defined in Eq.(15) is calculated with

$$
<O>=\frac{1}{N} \sum_{\mathbf{k}}\left[\cos \left(k_{x} n_{x}\right)+\cos \left(k_{y} n_{y}\right)\right] \cdot u(\mathbf{k}) \cdot v(\mathbf{k})
$$

These correlations were found to have a more rapid decay with distance than in a chain. The correlation length $\xi$ in a square lattice also decreases with both $\delta$ and $\alpha$ as shown in Fig.(12). There is a clear minimum in the correlation length at a certain $\alpha$ that shifts to higher values with $\delta$.

In summary, a simple linear spin wave theory brings out quite a few new features in ferrimagnetic systems under the combined effects of dimerization and frustration. The effects in both one- and two-dimensional ferrimagnetic 
systems are most visible in the elementary excitation spectra. Besides the critical value $\alpha_{c}$ of the frustration parameter at which the long range order is destroyed, there is another critical value $\alpha^{*}$ at which the elementary excitations undergo a mode softening, indicating a transition from a Neel-like to a spiral state. The LSWT shows that dimerization facilitates this transition. Both the critical values of $\alpha$ are $\delta$-dependent. While the ground state energy initially increases with increasing magnitude of frustration, it reaches a maximum and then decreases just before $\alpha$ reaches its critical value $\alpha_{c}$. Both sublattice magnetization and correlations decrease as the strength of dimerization and frustration increases, indicating the loss of order. The combined effects of dimerization and frustration in the case when dimerization is taken as $\frac{J_{1}}{1 \mp \delta}$ are quite different from the usual case $J_{1}(1 \pm \delta)$. The theory also shows that on a square lattice, dimerization initially opposes the transition to a spiral state, but then beyond a certain critical value $\delta_{c}$, the dimerization parameter facilitates the transition. In the case of a square lattice ferrimagnet, beyond a certain value $\delta^{*}$ of the dimerization parameter, the system loses long range Neel-like order before going through a transition to a spiral state. 


\section{References}

[*]E-mail: aiman_101@hotmail.com

[**]E-mail: ahnayyar@sat.net.pk

${ }^{1}$ S. Brehmer, H. -J. Mikeska, and S. Yamamoto, J. Phys. Condens. Matter 9, 3921 (1997).

${ }^{2}$ A. K. Kolezhuk, H. -J. Mikeska, and Sholi Yamamoto, Phys. Rev. B 55, R3336 (1997).

${ }^{3}$ Swapan K. Pati, S. Ramasesha, and Diptiman Sen, Phys. Rev. B 55, 8894 (1997); Swapan K. Pati, S. Ramasesha, and Diptiman Sen, J. Phys.: Condens. Matter 9, 8707 (1997).

${ }^{4}$ N. B. Ivanov, Phys. Rev. B 57, R14024 (1998).

${ }^{5}$ Aiman Al-Omari and A. H. Nayyar, J. Phys.: Condes. Matter 11, 465 (1999).

${ }^{6}$ N. B. Ivanov, J. Richter, and U. Schollwock, Phys. Rev. B 58, 14456 (1998).

${ }^{7}$ Shoji Yamamoto, Takahiro Fukui, and Tôru Sakai, cond-mat/0001004 (2000); Shoji Yamamoto, cond-mat/0004297 (2000).

${ }^{8}$ Shoji Yamamoto, S. Brehmer, and H. J. Mikeska, Phys. Rev. B 57, 13610 (1998).

${ }^{9}$ Shoji Yamamoto and Tôru Sakai, J. Phy. Soc. Jpn 67, 3711 (1998).

${ }^{10}$ Shoji Yamamoto, and Takahiro Fukui, Phys. Rev. B 57, 14008 (1998).

${ }^{11}$ Shoji Yamamoto, Phys. Rev. B 59, 1024 (1999).

${ }^{12}$ Shoji Yamamoto, Takahiro Fukui, Klaus Maisinger, and Ulrich Schollock, J. Phys.: Condes. Matter 10, 11033 (1998).

${ }^{13}$ For more details see Ref. 
${ }^{14}$ It was assumed? 3 as well as shown explicitly l $^{3}$ earlier that the behavior of the an alternating spin chain is similar regardless of the values of $s_{1}$ and $s_{2}$.

${ }^{15}$ R. Chitra, Swapan Pati, H. R. Krishnamurthy, Diptman Sen, and S. Ramasesha, Phys. Rev. B 52. 6581 (1995).

${ }^{16}$ Sanyee Tang, and J. E. Hirsch, Phys. Rev. B 37, 9546 (1988).

${ }^{17}$ A. Feiguin, C. J. Gazza, A. E. Trumper, and H. A. Ceccatto, J. Phys.: Condens. Matter 6, L503 (1994). 


\section{Figure captions}

Figure 1: Dependence of the critical frustration parameter $\alpha_{c}$ on the dimerization parameter $\delta$ for the one-dimensional spin systems $\left(1, \frac{1}{2}\right),\left(\frac{3}{2}, 1\right)$ and $\left(\frac{3}{2}, \frac{1}{2}\right)$. This is for the case when the dimerization dependence of the nearest neighbor interaction is taken as $J^{ \pm}=J_{1}(1 \pm \delta)$.

Figure 2: The ground state energy $\varepsilon_{g}$ of the alternating spin chain $\left(1, \frac{1}{2}\right)$ vs the frustration parameter $\alpha$ for different values of $\delta$ for $J^{ \pm}=J_{1}(1 \pm \delta)$. The curve for each $\delta$ terminates at the respective $\alpha_{c}$. The maximum in the ground state energy occurs at an $\alpha$ that shifts to lower values with higher $\delta$. In the dimer limit $(\delta \rightarrow 1)$, the energy monotonically decreases with $\alpha$, a feature peculiar to the combined effect of dimerization and frustration.

Figure 3: The elementary excitation spectra for the chain $\left(1, \frac{1}{2}\right)$ for various values of the frustration parameter; (a) for $\delta=0.0$, (b) $\delta=0.4$ and (c) $\delta=0.8$. This is for $J^{ \pm}=J_{1}(1 \pm \delta)$ for which the $k=0$ optic mode is $\delta-$ and $\alpha$-independent. With nonzero $\alpha$, the difference between the two modes is no longer constant. Softening of the two modes for $\alpha$ beyond $\alpha^{*}$ is easily discernible. The other two spin systems $\left(\frac{3}{2}, 1\right)$ and $\left(\frac{3}{2}, \frac{1}{2}\right)$ show the same schematic behavior.

Figure 4: Sublattice magnetizations for the chain $\left(1, \frac{1}{2}\right)$ as functions of $\alpha$ and $\delta$. The curves terminate at the respective values of $\alpha_{c}$. The behavior is schematically the same for the other two spin systems.

Figure 5: The variation of the correlation length $\xi$ for the alternating spin chain $\left(1, \frac{1}{2}\right)$ vs frustration parameter $\alpha$, for different values of $\delta$ when $J^{ \pm}=J_{1}(1 \pm \delta)$. The curves stop short of the respective critical value $\alpha_{c}$ because of the strong fluctuations that $\xi$ experiences near this point. The results are schematically the same for the other two spin systems.

Figure 6: The dependence of $\alpha_{c}$ on dimerization parameter when $J^{ \pm}=$ $\frac{J_{1}}{1 \mp \delta}$ for the three spin chains. This is to be seen in contrast to Fig.(1) where the variation is shown for the other choice of $J^{ \pm}$.

Figure 7: The ground state energy $\varepsilon_{g}$ for the alternating spin chain $\left(1, \frac{1}{2}\right)$ as a function of the frustration parameter $\alpha$ for different values of $\delta$ for the case when $J^{ \pm}=\frac{J_{1}}{1 \mp \delta}$. Like in the case of $J^{ \pm}=J_{1}(1 \pm \delta)$ the energy has 
a maximum at an $\alpha$ that decreases with $\delta$. Again, in the limit $\delta \rightarrow 1$, the ground state energy decreases monotonically with $\alpha$. The other two spin systems show the same schematic behavior. In accordance with the peculiar dependence of $\alpha_{c}$ on $\delta$, as in Fig.(6), the curves for higher $\delta$ can go up to higher values of $\alpha$.

Figure 8: The elementary excitation spectra for the chain $\left(1, \frac{1}{2}\right)$ for various values of the frustration parameter and for; (a) $\delta=0.0$, (b) $\delta=0.4$ and (c) $\delta=0.8$. This is for the case with $J^{ \pm}=\frac{J_{1}}{1 \mp \delta}$ for which the optic mode at $k=0$ is $\delta$-dependent. Because $\alpha_{c}$ in this case remains constant over a larger range of $\delta$, the modes in all the three figures are plotted up to just before the critical value $\alpha_{c}$. The mode softening is as explained in the text. The other spin systems have the same schematic behavior..

Figure 9: The elementary excitation dispersion relations of the ferrimagnetic system $\left(1, \frac{1}{2}\right)$ on a square lattice. The spectra are shown for different $\alpha$ and for (a) $\delta=0.0$, (b) $\delta=0.4$ and (c) $\delta=0.8$.

Figure 10: The dependance of $\alpha^{*}$ and $\alpha_{c}$ on the dimerization parameter $\delta$ for the three spin systems on square lattice. The value of $\delta$ at which the peak occurs is independent of the spin components of a ferrimagnetic system. For $\delta>\delta^{*}$ the long range order is destroyed before the transition to a spiral state can take place.

Figure 11: The $\alpha-$ and $\delta$ - dependence of the staggered magnetization of a square lattice $\left(1, \frac{1}{2}\right)$ ferrimagnet. The same schematic behavior is shown by the other spin systems.

Figure 12: Correlation length $\xi$ vs frustration parameter $\alpha$ for different values of the dimerization parameter $\delta$ in a square lattice $\left(1, \frac{1}{2}\right)$ ferrimagnet. 
Fig(1)

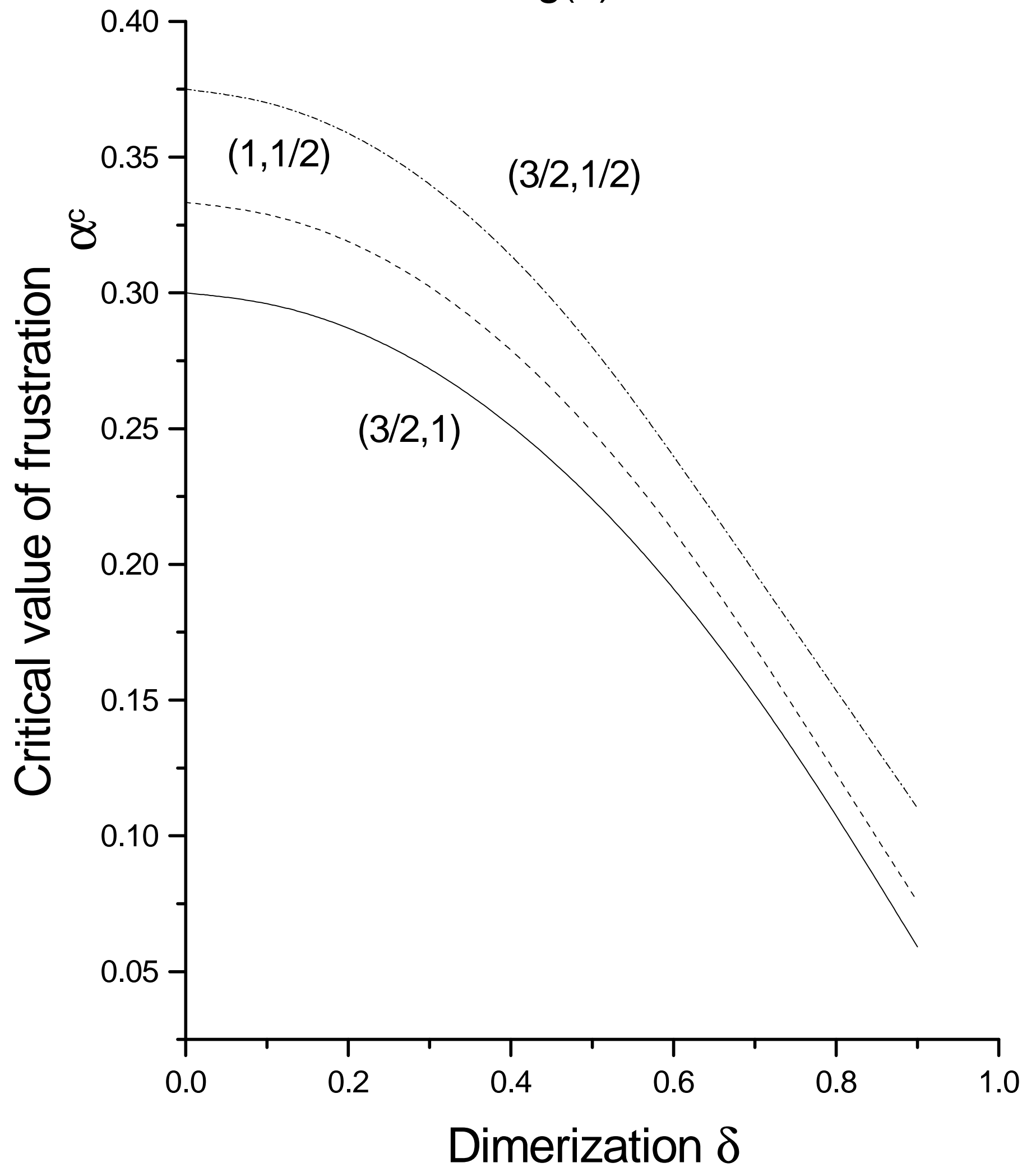


Fig.(2)

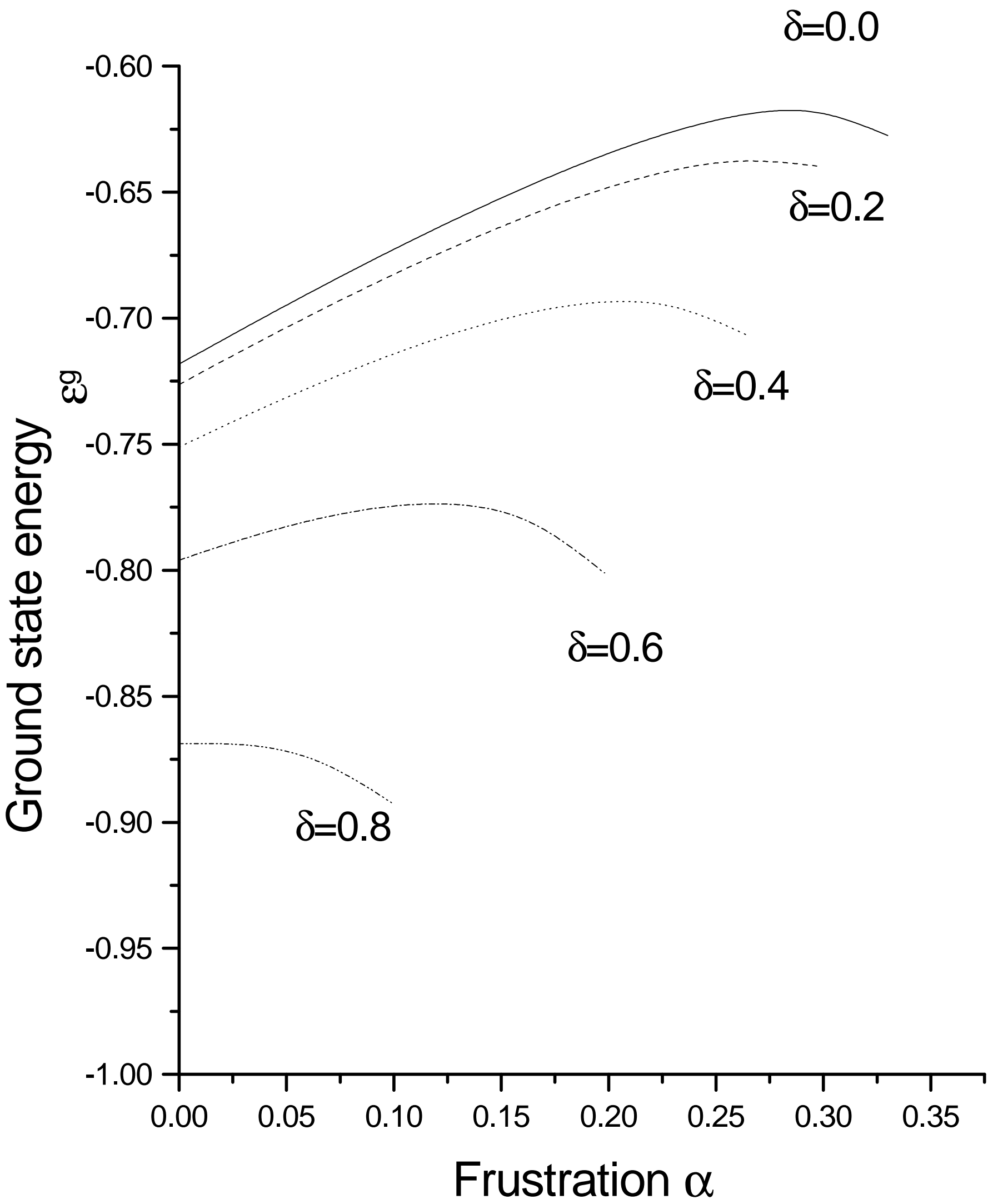




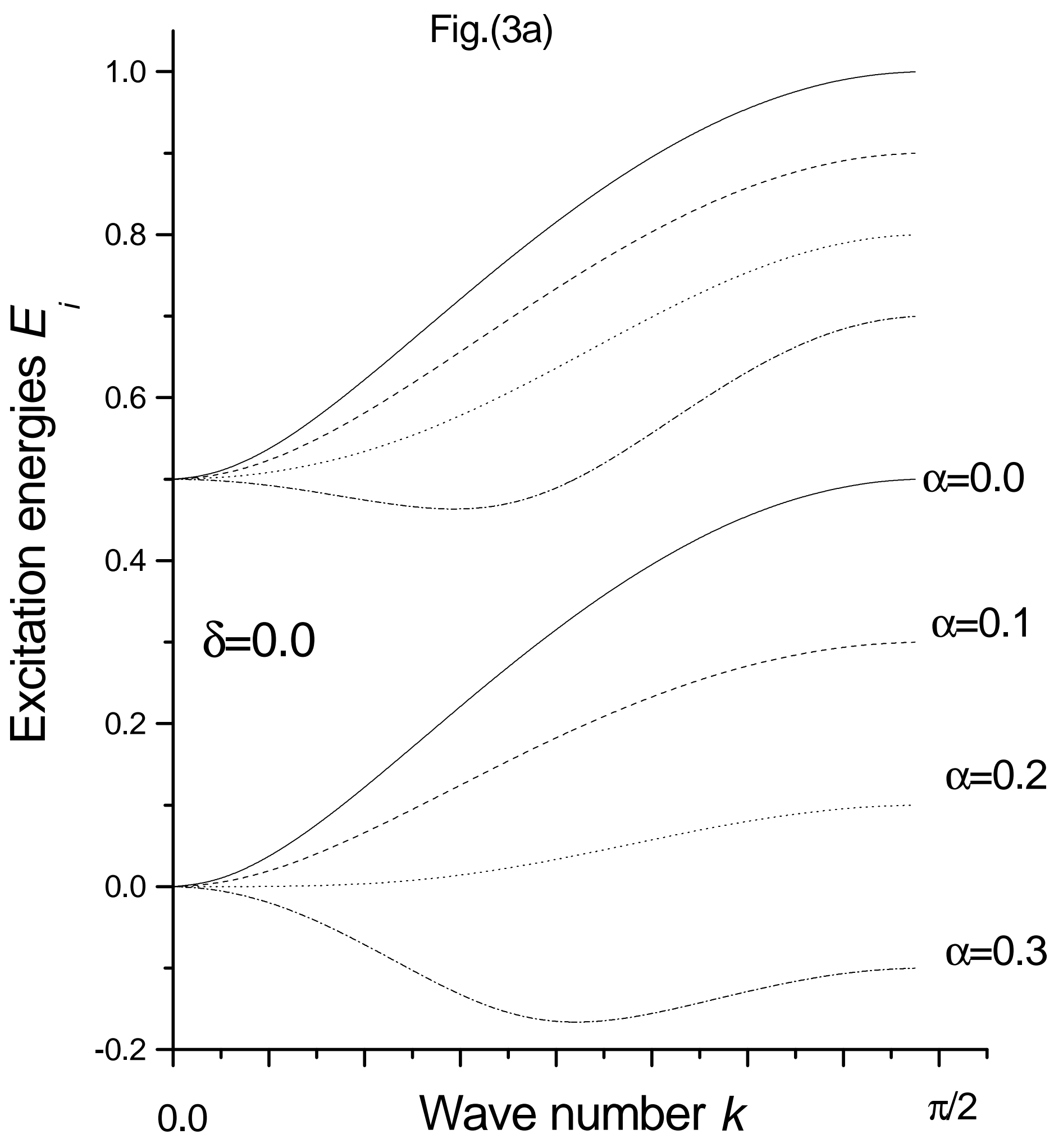


Fig.(3b)

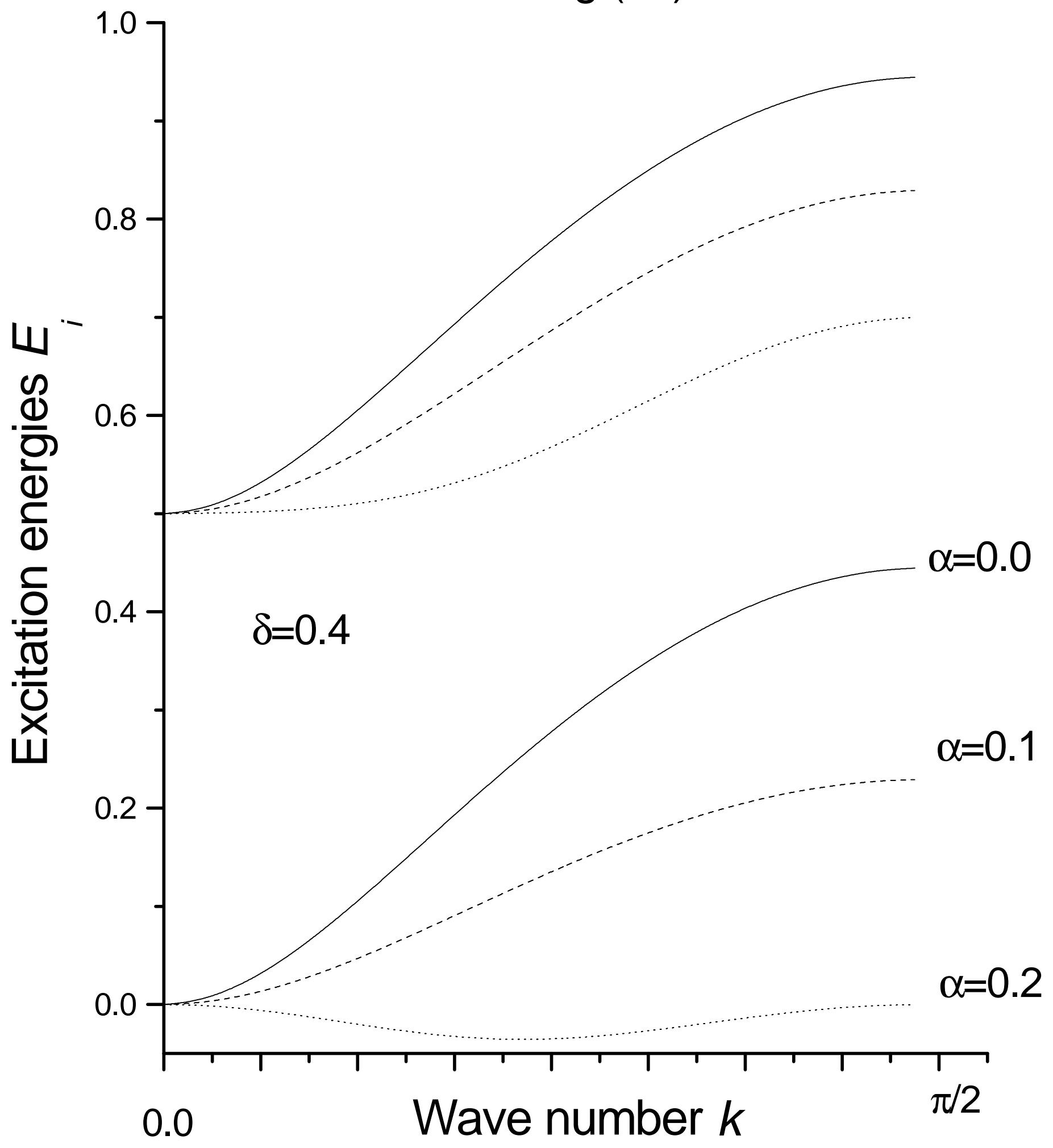


Fig.(3c)

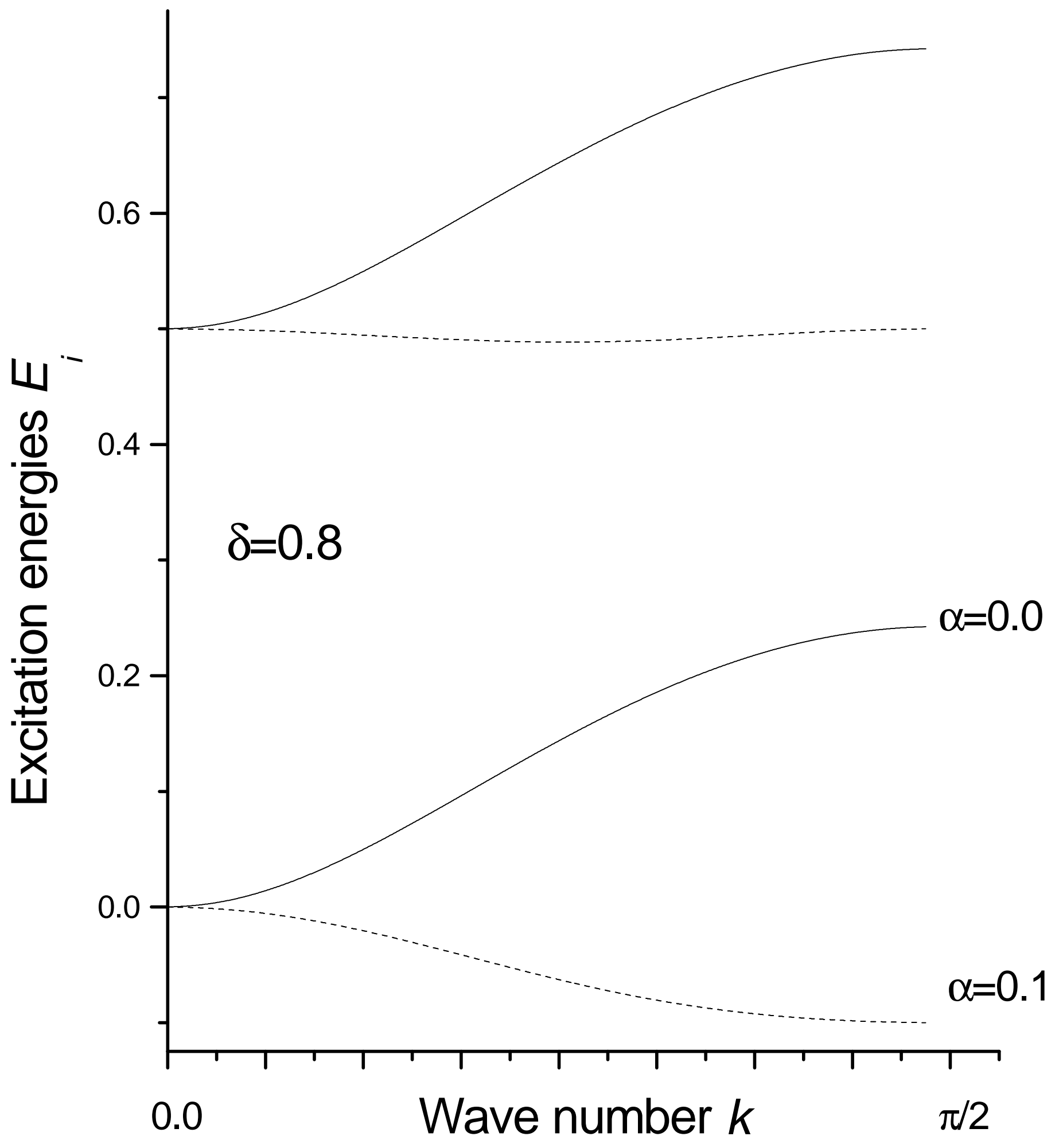




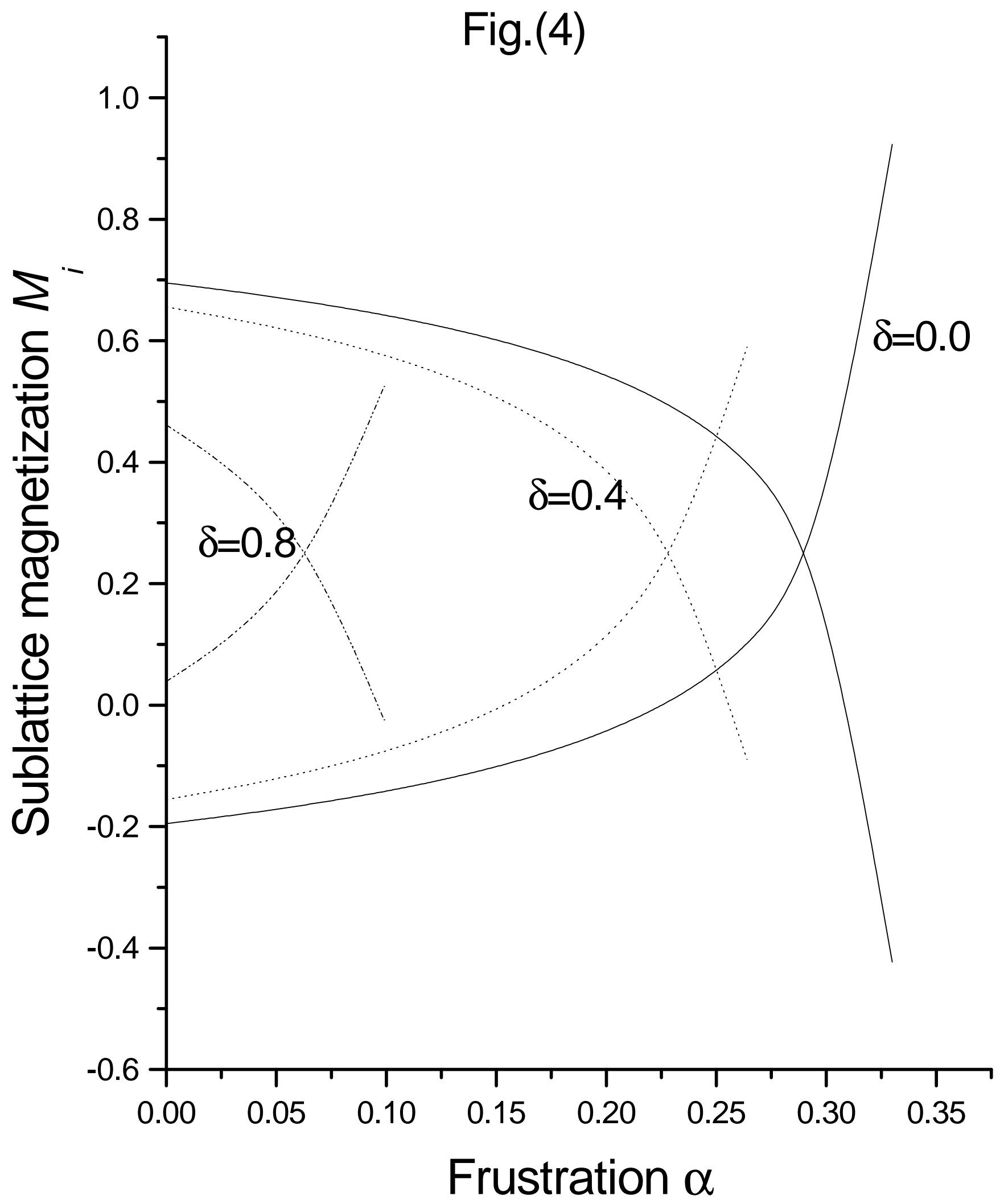


Fig.(5)

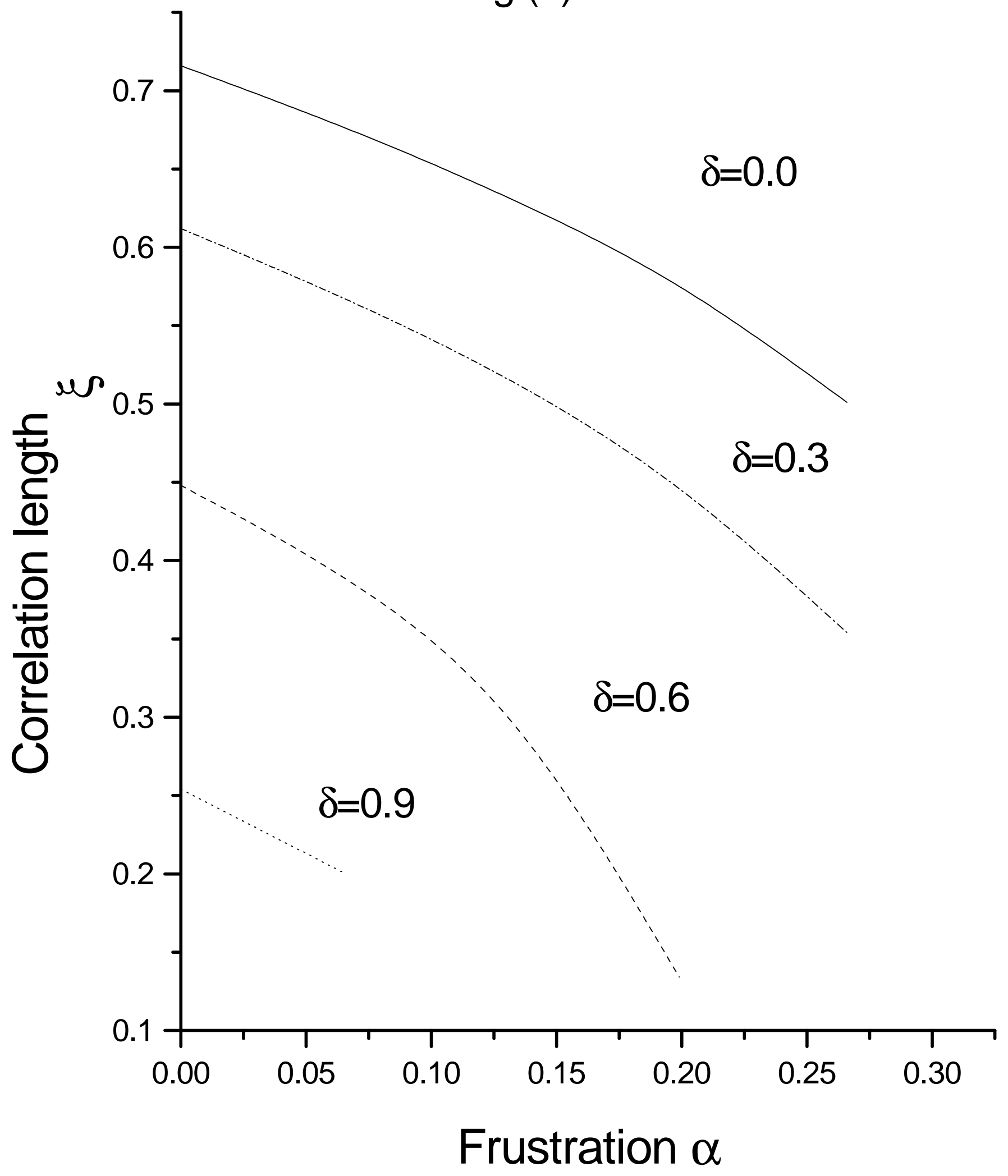




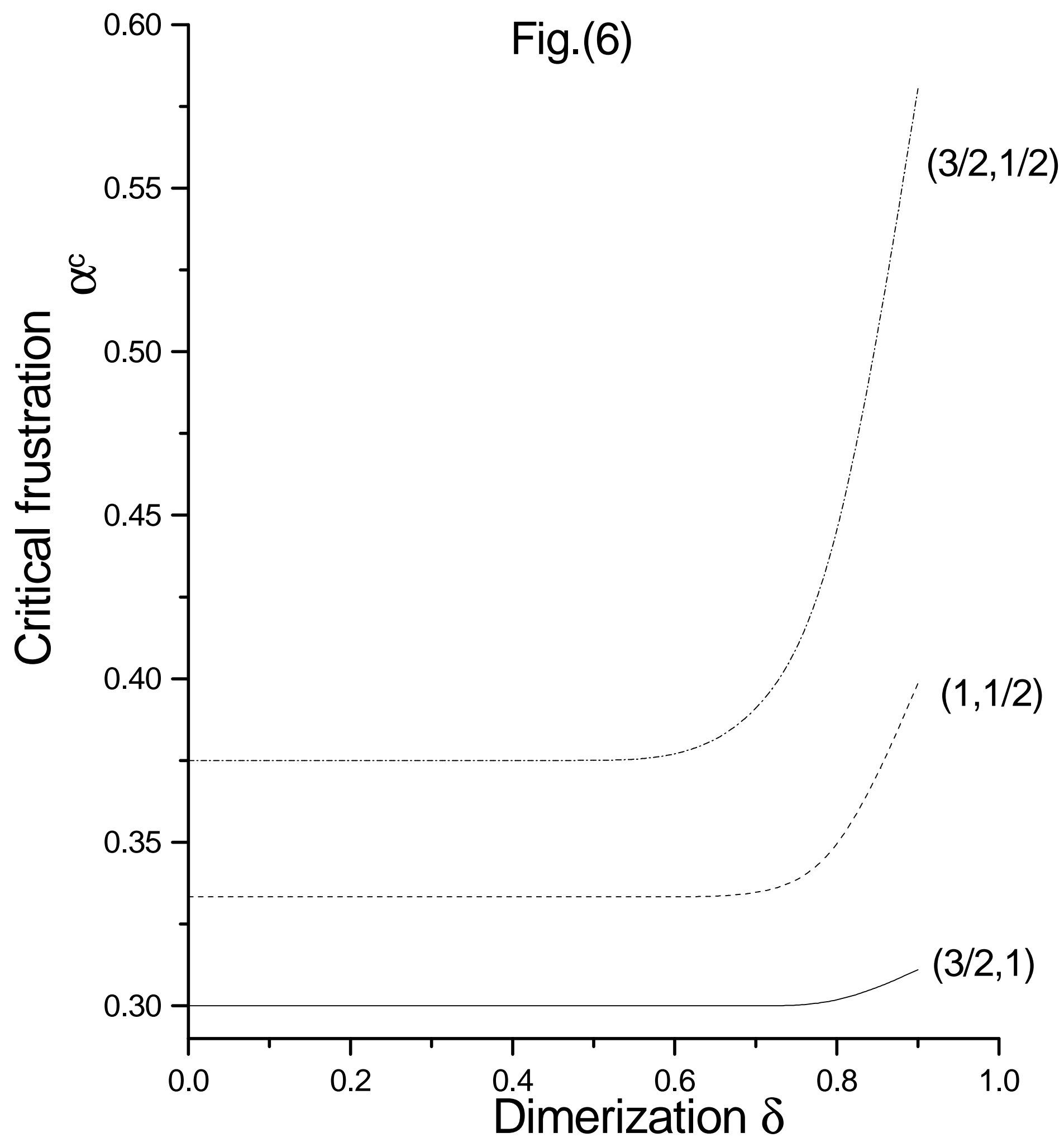


Fig.(7)

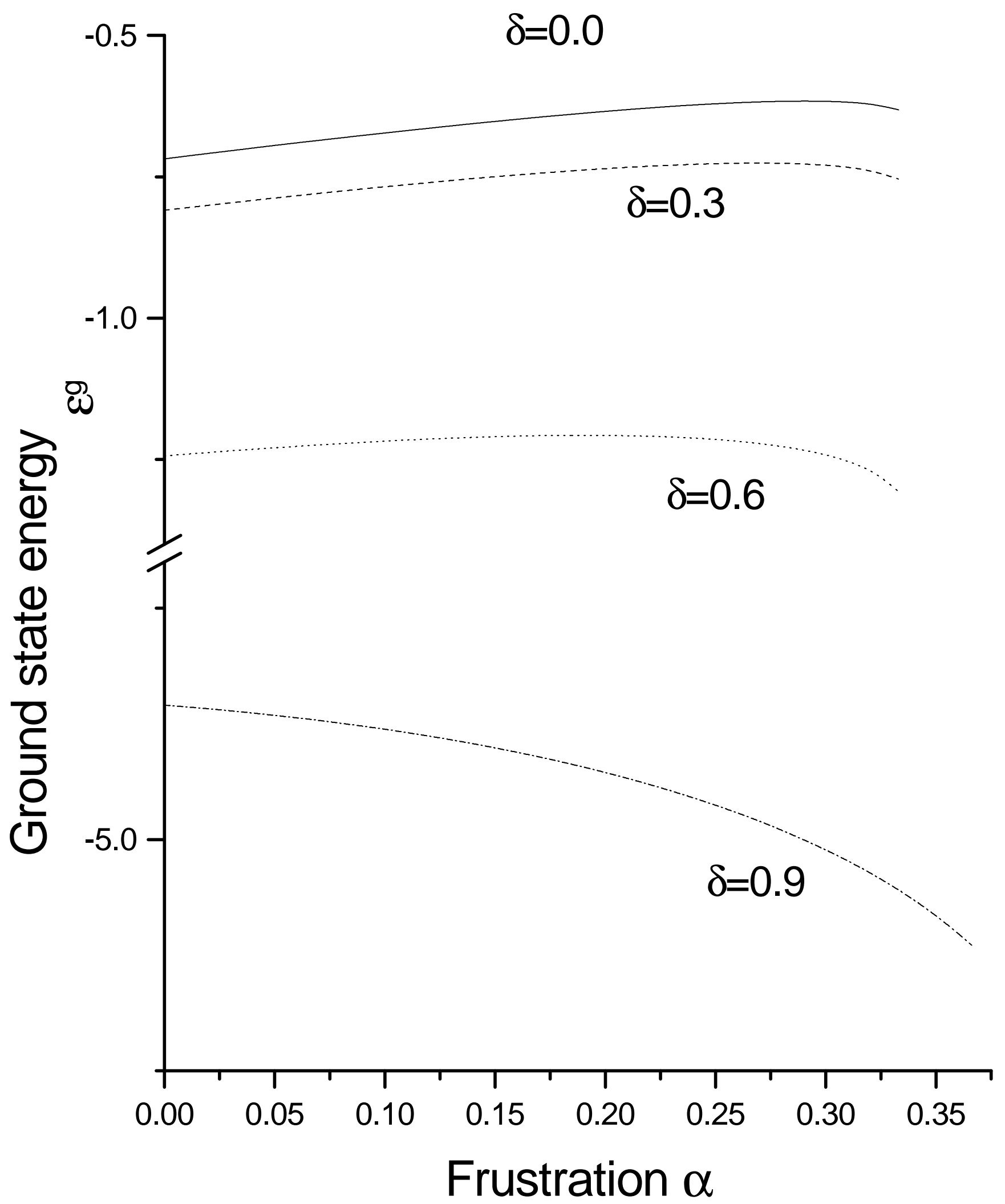


Fig.(8a)

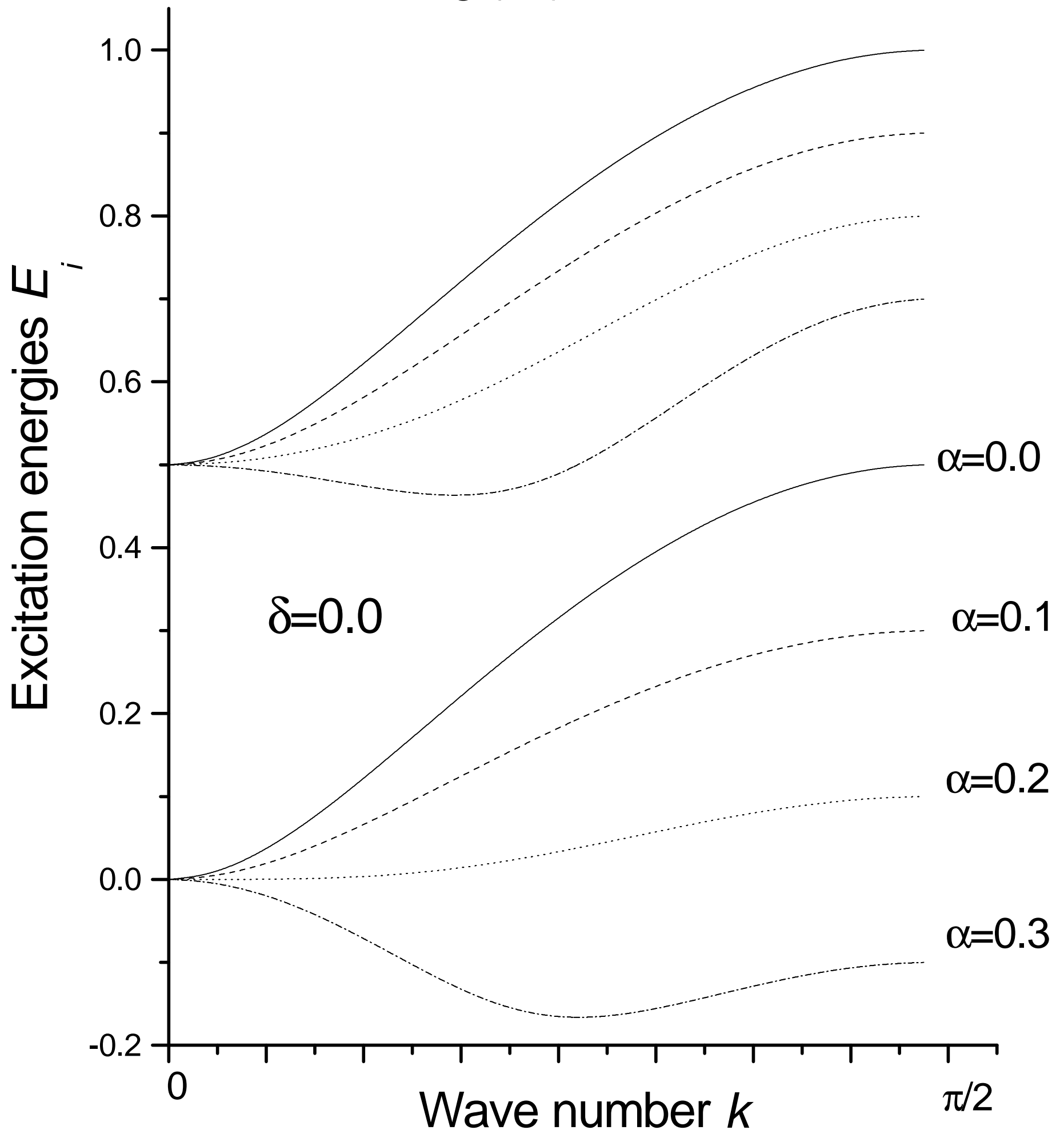


Fig.(8b)

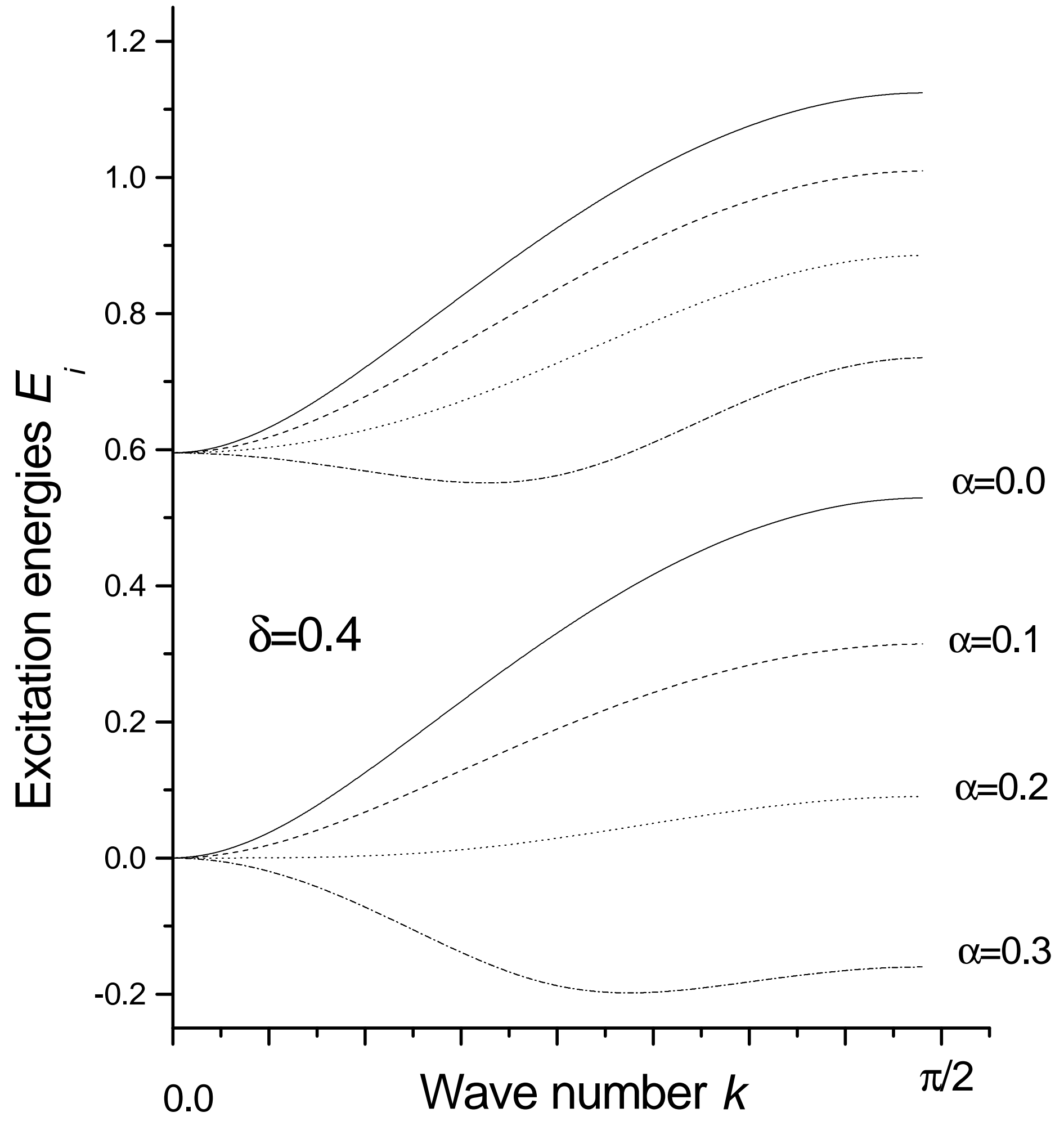


Fig.(8c)

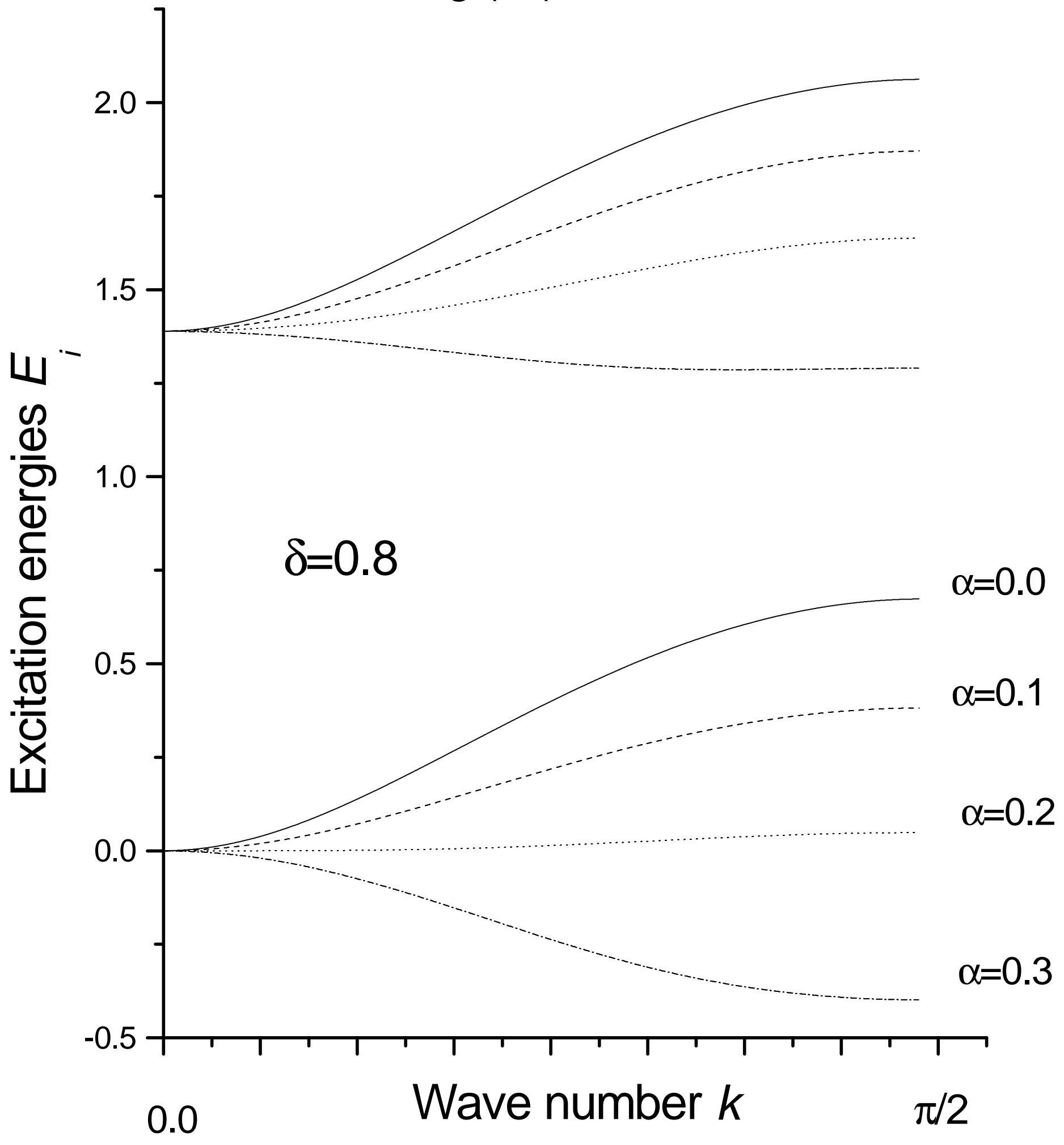


Fig.(9a)

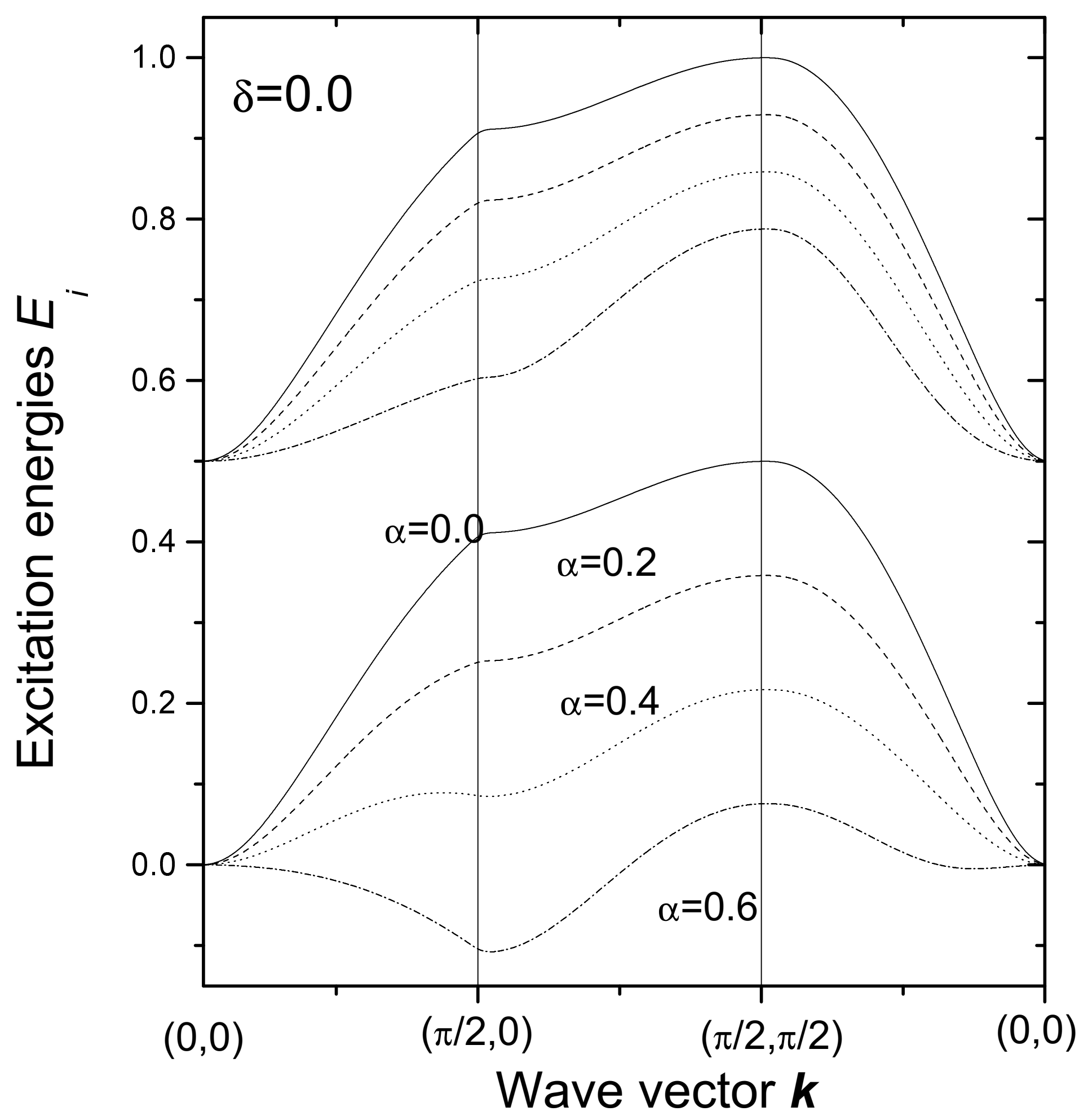


Fig.(9b)

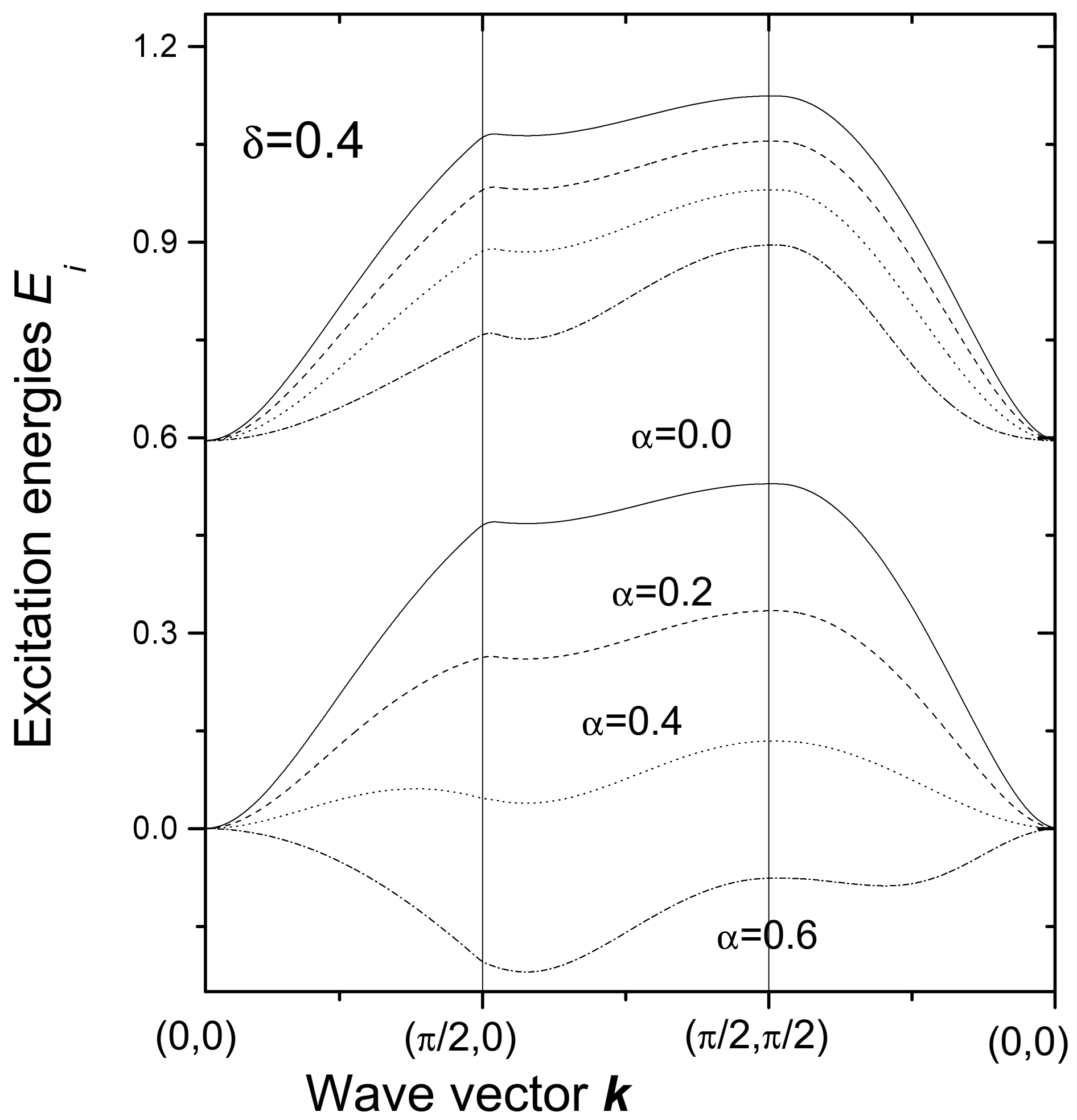


Fig.(9c)

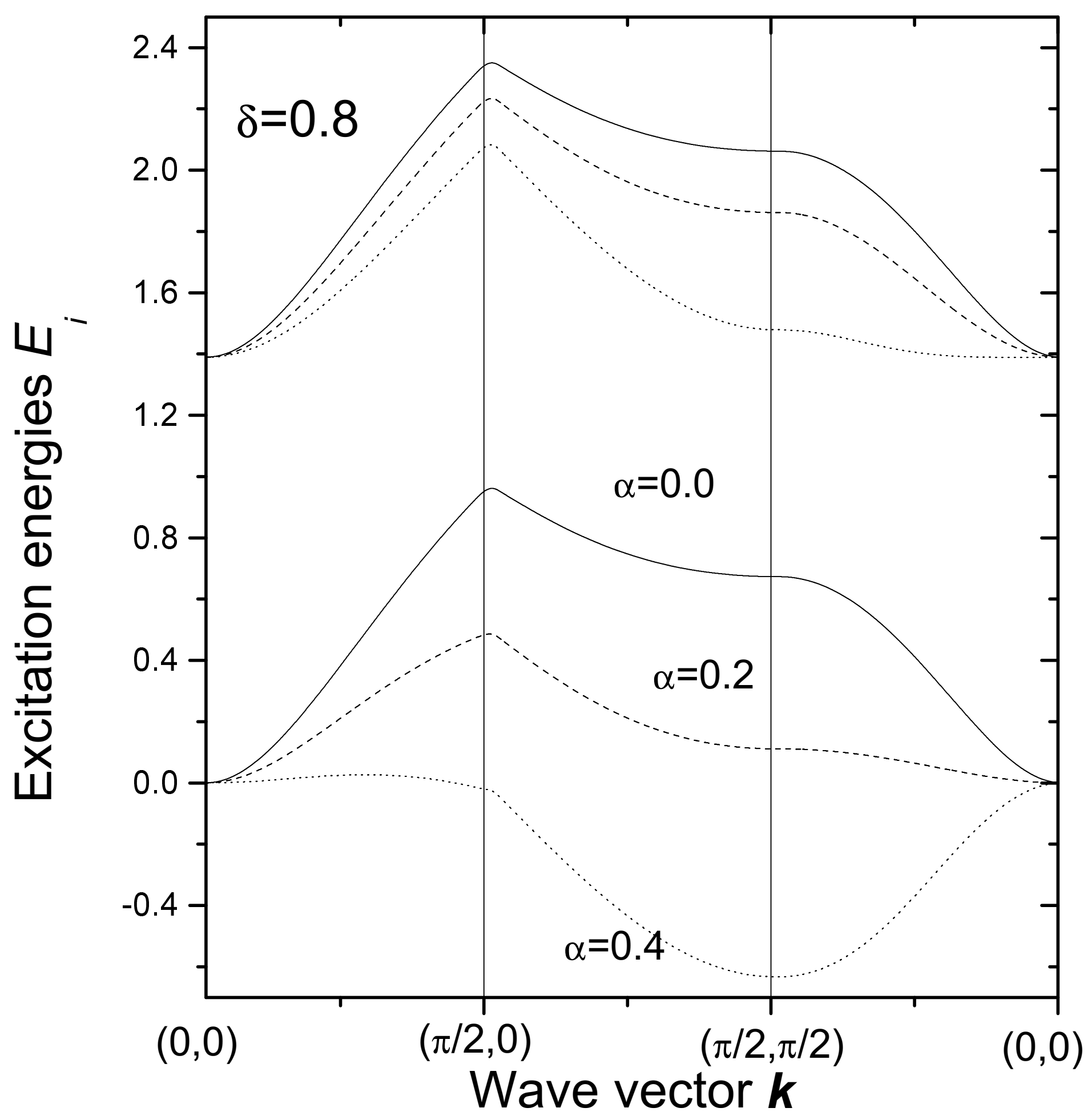


Fig.(10)

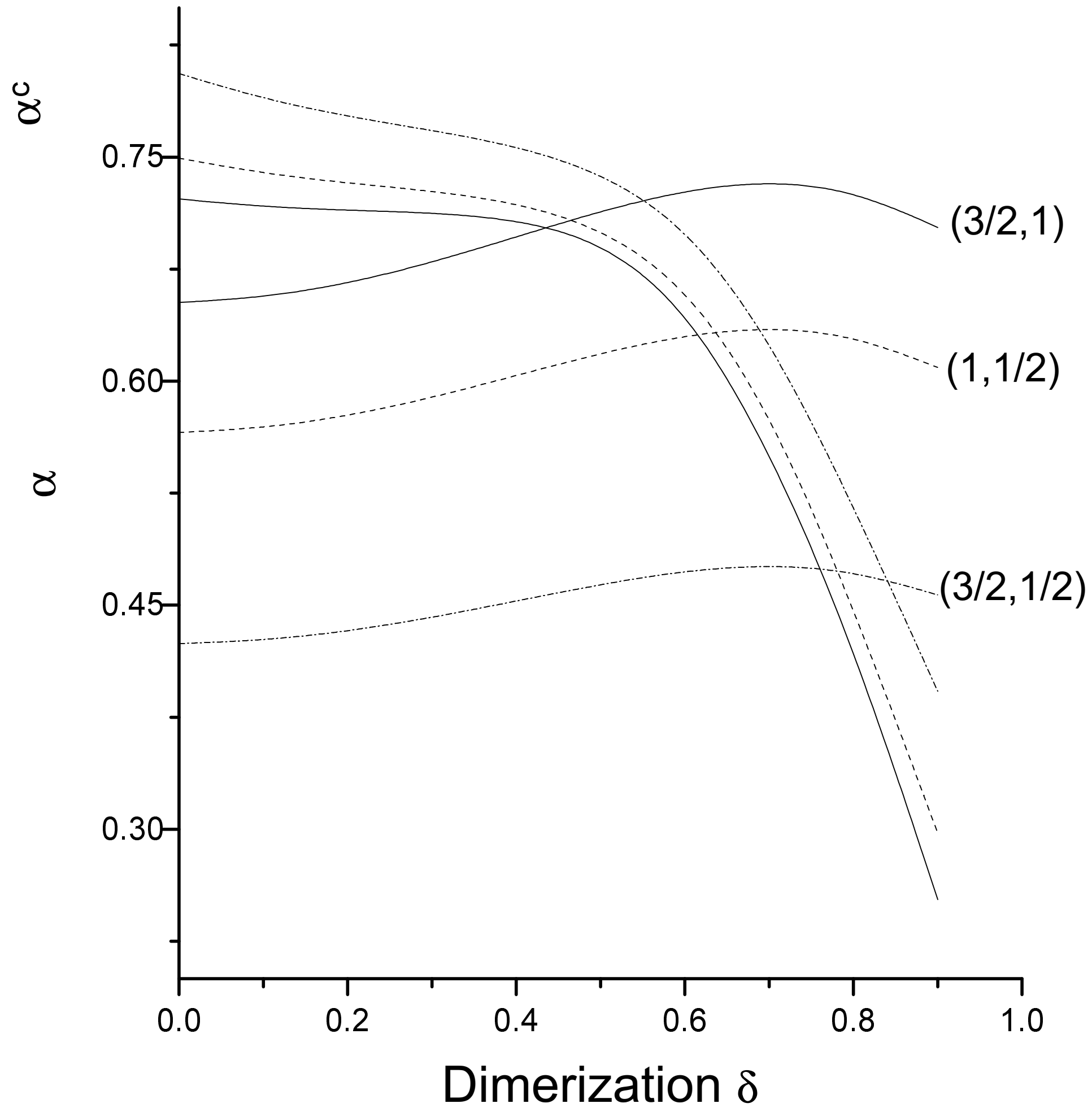


Fig.(11)

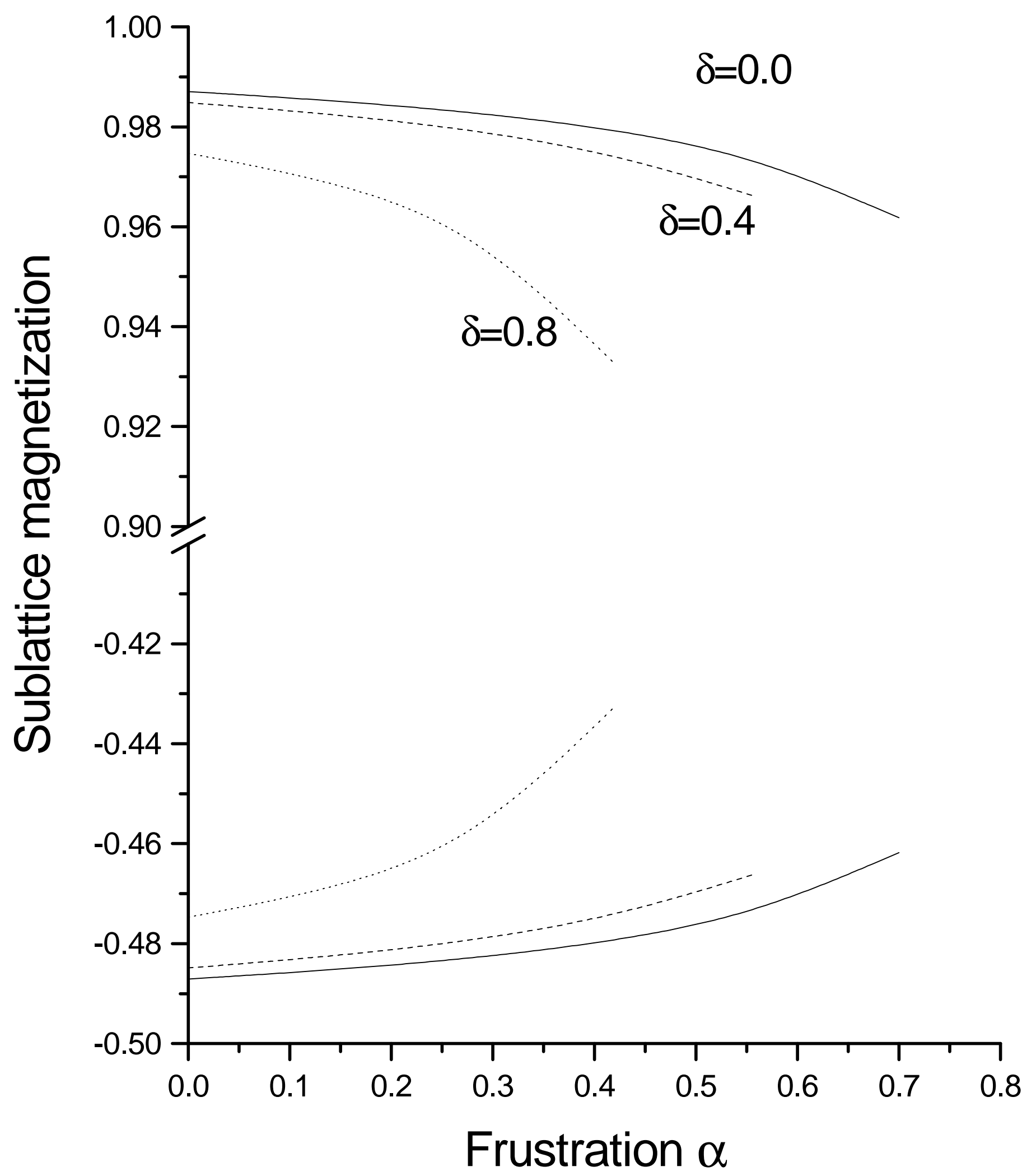


Fig.(12)

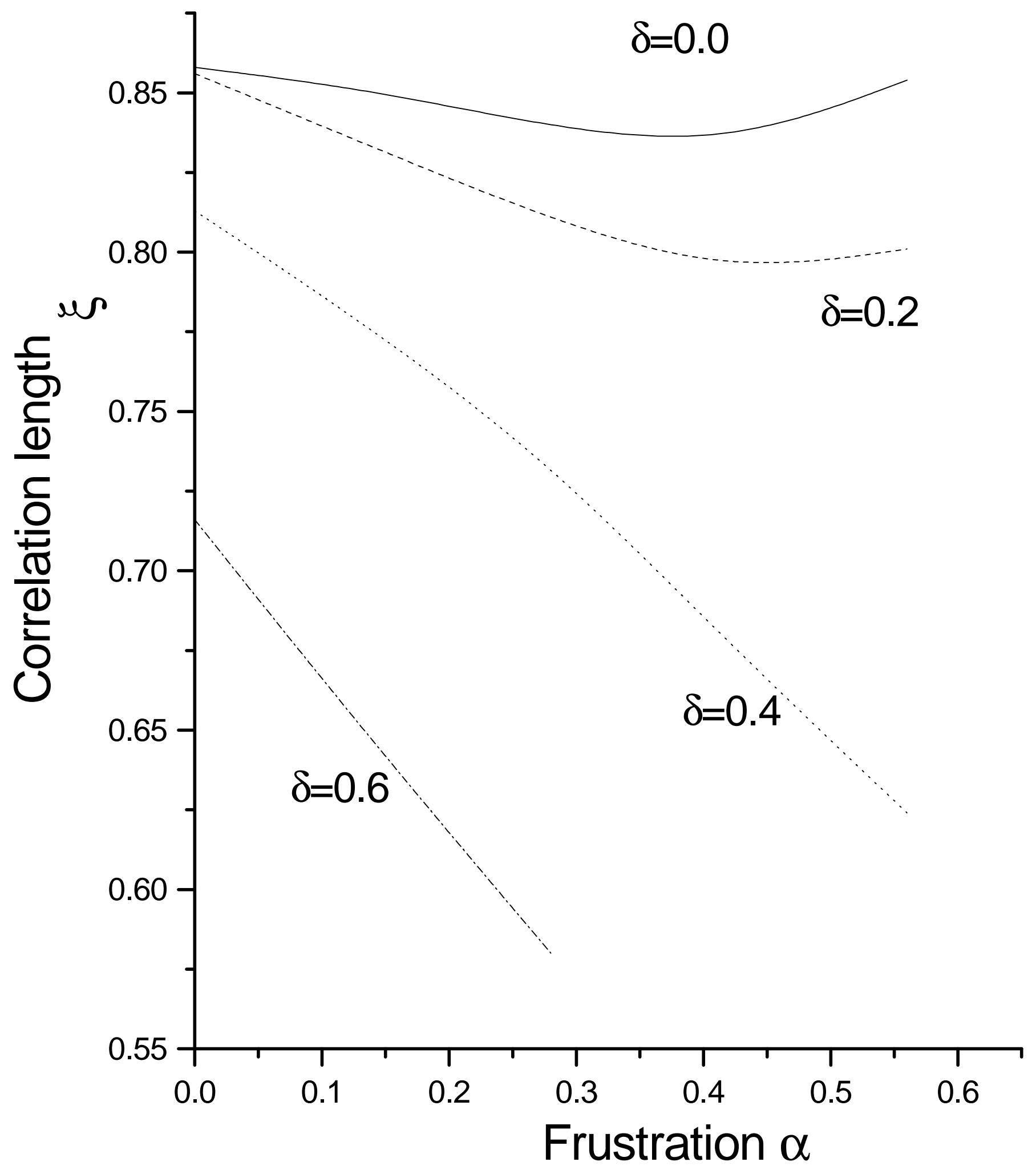

